\title{
Mobile Image Ratiometry: A New Method for Instantaneous Analysis of Rapid Test Strips
}

\author{
Donald C. Cooper ${ }^{1,2}$, Bryan Callahan'2, Phil Callahan ${ }^{2} \&$ Lee Burnett ${ }^{2}$
}

Here we describe Mobile Image Ratiometry (MIR), a new method for the automated quantification of standardized rapid immunoassay strips using consumer-based mobile smartphone and tablet cameras. To demonstrate MIR we developed a standardized method using rapid immunotest strips directed against cocaine (COC) and its major metabolite, benzoylecgonine (BE). We performed image analysis of three brands of commercially available dye-conjugated anti-COC/BE antibody test strips in response to three different series of cocaine concentrations ranging from 0.1 to $300 \mathrm{ng} / \mathrm{ml}$ and $B E$ concentrations ranging from 0.003 to $0.1 \mathrm{ng} / \mathrm{ml}$. These data were then used to create standard curves to allow quantification of COC/BE in biological samples. MIR quantification of $\mathrm{COC}$ and BE proved to be a sensitive, economical, and faster alternative to more costly methods, such as gas chromatography-mass spectrometry, tandem mass spectrometry, or high pressure liquid chromatography. MIR is a valuable tool that provides instant data acquisition, tracking and analysis for the emerging field of mobile platform informatics (MPI) and smartphone informatics (SPI).

\section{RESULTS}

Cocaine and benzylecgonine standard curves

Each $\mathrm{COC}$ and $\mathrm{BE}$ standard provided colored signal bands that were quantified and used to create a standard curve. For the test strips obtained from Craig Medical, an exponential function provided the best fitting curve for both the COC and $\mathrm{BE}$ data. Sensitivity for $\mathrm{COC}$ ranged from 3 to $30 \mathrm{ng} / \mathrm{ml}$, whereas sensitivity for BE ranged from 0.003 to $0.1 \mathrm{ng} / \mathrm{ml}$. Thus, the Craig Medical test strips were 250 times more sensitive towards BE than COC. Cocaine sensitivity for Medimpex test strips ranged from 0.1 to $2 \mathrm{ng} / \mathrm{ml}$, whereas sensitivity for Q Test strips ranged from 5 to $100 \mathrm{ng} / \mathrm{ml}$. Thus, the Medimpex test strips were approximately 10 times more sensitive to cocaine compared to those from Craig Medical and the $\mathrm{Q}$ Test strips approximately 3 times less sensitive to cocaine compared to those from Craig Medical. MIR analysis produced fast, repeatable and highly sensitive detection of COC and BE.

\section{DISCUSSION}

In this paper, we describe MIR, which uses low cost immunoassay strips, a smart phone or tablet computer camera, and automated image analysis to detect and quantify cocaine and benzoylecgonine. MIR has many possible applications when and can be used for almost any number of immunoassay test strips. Many immunoassay test strips exist which test for anything from drugs of abuse to water contaminants and infectious agents, such as bacteria or parasites. Foremost, MIR represents a powerful tool for use in developing countries where resources and trained personnel are limited and immunoassay test strips and cell phones are relatively inexpensive and require little training. Results can be photographed by individuals, transmitted to a central server for archiving and analysis, and the results sent back within minutes. Smart phones and tablet computers can automatically tag photos with coordinates, allowing end-users to track results geographically. The development of MIR (Mobile Assay Inc., www.mobileassay.com) is one example that reflects the advancement in the field of Mobile Platform Informatics (MPI), which includes tablets and smart phones. New smart tools for MPI are advancing as mobile devices develop new capability to capture and quantify information previously acquired through costly specialized equipment. In the future it is anticipated that these tools will allow low-cost consumer-based devices to serve as multifunctional data testing, tracking and analyzing devices with applications in a variety of industries.

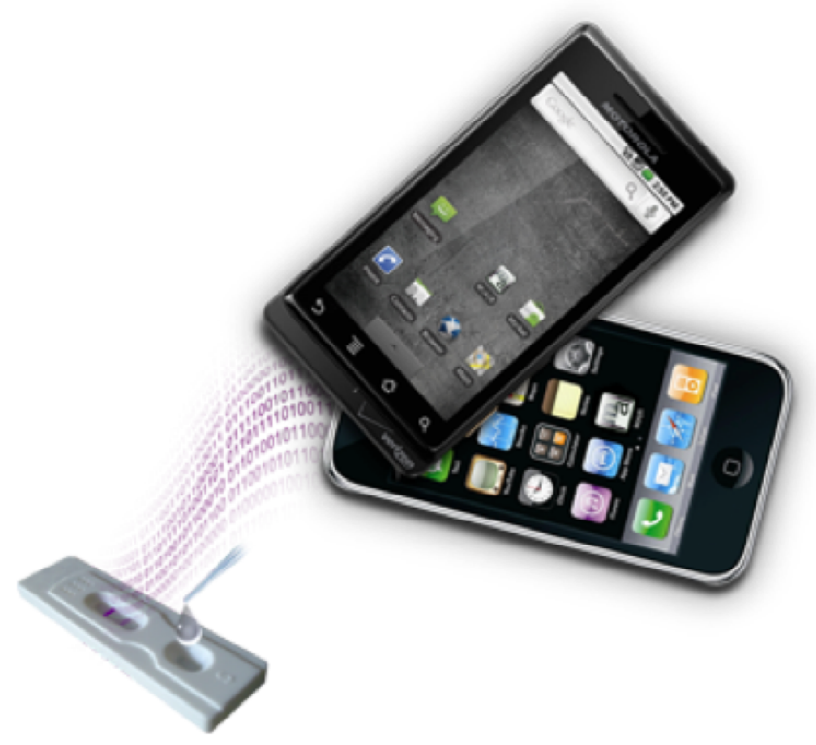

Fig 1. MIR acquisition and analysis of rapid test strips on the Android and iOS platforms.

1.Institute for Behavioral Genetics/Department of Psychology and Neuroscience, University of Colorado, Boulder. 148030 th St. Boulder, Co 80303.

2.Mobile Assay Inc., www.mobileassay.com1

*dcooper@colorado.edu 


\section{Brief Report}
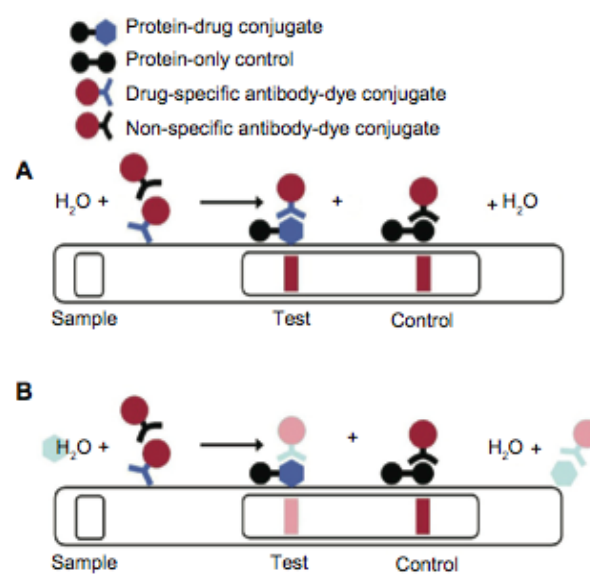

C
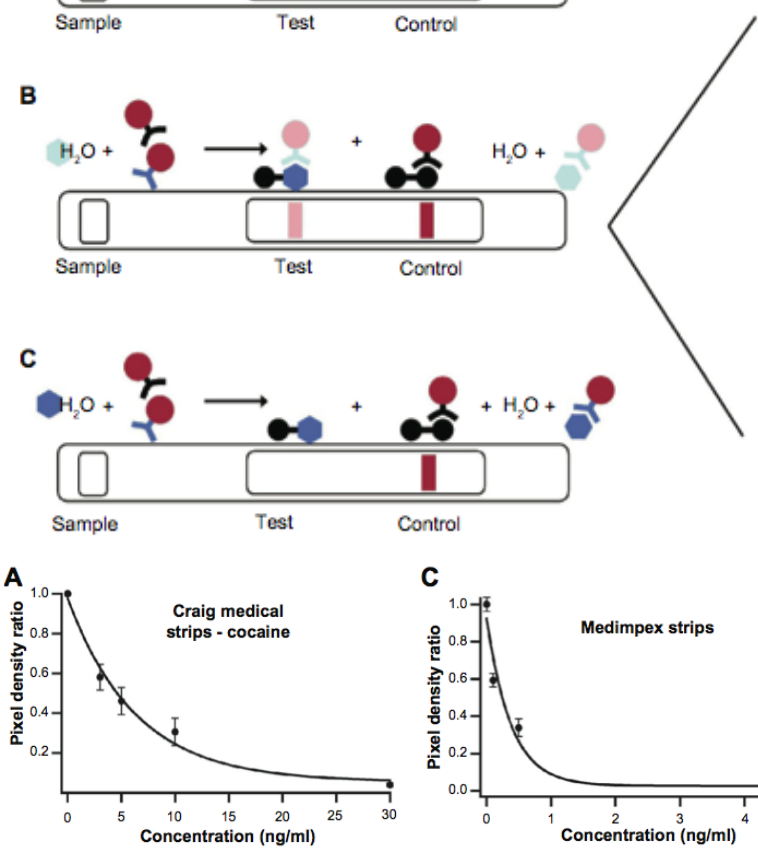

C
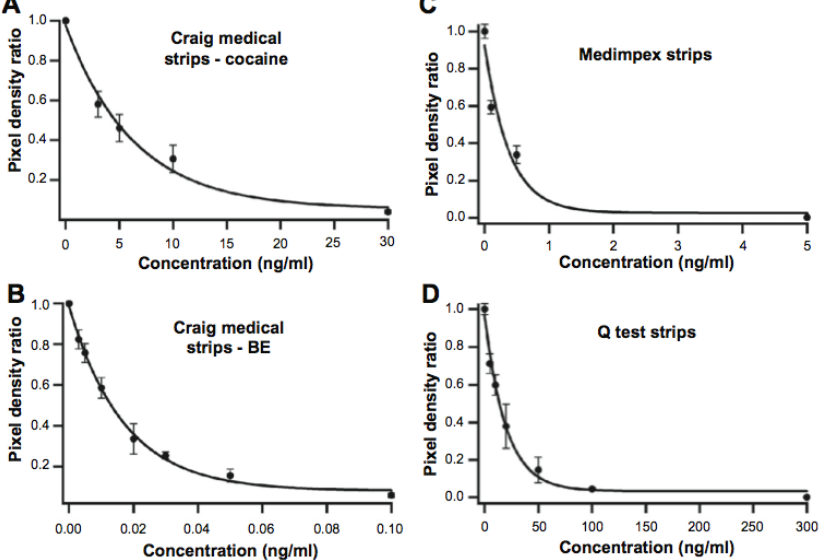

Fig 3. Craig medical, medimpex, and Q test standard curves. A) To allow quantification of cocaine and $\mathrm{BE}$ in unknown samples, standard curves were created. First, a series of COC standards $(0,3,5,10$, and $30 \mathrm{ng} / \mathrm{ml})$ were created and applied to test strips obtained from Craig Medical in triplicate. B) To determine if the test strips have the same sensitivity towards COC and $\mathrm{BE}$, a series of $\mathrm{BE}$ standards $(0,0.003,0.005,0.01,0.02,0.03,0.05$, and 0.1 $\mathrm{ng} / \mathrm{ml}$ ) were created and applied to Craig Medical test strips in triplicate. Results were obtained and plotted as in a. Craig Medical Test strips were able to detect BE levels approximately 300 times lower than that of cocaine. C) Different brands may have different sensitivities to $\mathrm{COC} / \mathrm{BE}$. To test this, strips were obtained from Medimpex United, Inc. Four COC standards $(0,0.1,0.5$, and $5 \mathrm{ng} / \mathrm{ml}$ ) were created and applied to test strips in triplicate. Results were quantified and plotted as in A. Medimpex test strips were approximately 30 times more sensitive to cocaine than the Craig Medical test strips. D) As in C, except using Q Test strips and seven COC standards $(0,5,10,20,50,100$, and $300 \mathrm{ng} / \mathrm{ml}$ ). Results indicate that Craig Medical test strips are approximately 3 times more sensitive to cocaine than Q Test strips. Adapted from Figure 3 in Substance Abuse: Research and Treatment 2010:4.

\section{METHODS}

Generation of Cocaine and Benzoylecgonine Standard curves

In order to quantify $\mathrm{COC} / \mathrm{BE}$ levels a series of known concentrations were made to generate a standard curve. Unknown samples may be compared to the standard curve, which allows quantification. See www.neuro-cloud.net for detailed results.
Fig 2. Test strip immunoassay system. A) Liquid samples are placed on the test strip sample area, where capillary action draws the fluid along with dye conjugated antibodies across a test area and control area. If no drugs (blue hexagons) are present, dye will bind at both locations, causing two bands to appear. B) At intermediate doses, drug-dye binding is incomplete, resulting in a light test band which varies in intensity according to the drug concentration. A camera and image analysis software can then quantify the relative intensity of the two bands. c) If drugs are present at sufficient concentrations, they completely bind the drug specific dye and prevent a band from appearing in the test area at all. Adapted from Figure 1 of Cadle et al., 2010 Substance Abuse: Research and Treatment 2010:4.

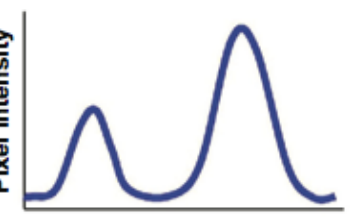

\section{Automatic web based quantification}

The ability to transmit an image from a wireless or cellular device and receive results instantly is crucial for an effective mobile diagnostic tool. To that end, we created MIR analysis, a patent pending application that automatically reports colloidal gold signal on standard immunotest strips. The MIR subtracts background noise, selects the signal bands, plots the pixel density ratio of the bands and measures the area underneath each peak. The result is immediately reported on the mobile device and if necessary they are sent to a secure cloud-based server for further analysis and storage. See www.neuro-cloud.net for detailed analysis.

\section{Testing background illumination}

Photos of test strips may be taken under many different lighting conditions. We tested this by applying ddH20 to Craig Medical test strips and taking images using the Sprint HTC 3.2 Megapixel camera phone at 1 hour at various levels of background illumination. The luminosity $(51,75,100$, and 154 average luminosity) was determined using Adobe Photoshop CS3, and signal bands were quantified as described above. See www.neuro-cloud.net for detailed analysis.

\section{ACKNOWLEDGEMENTS}

We would like to thank Leah Leverich, Ph.D. for her continued technical assistance.

Protected under creative commons attribution licence, learn more at http://www.creativecommons.com 